\title{
Decision Support Systems for Predicting Erectile Dysfunction
}

\section{Hsuan-Hung LIN ${ }^{1,}$, Po-Chou CHAN ${ }^{1,}$, Ming-Huei LEE ${ }^{1,3}$, Yung-Fu $\mathrm{CHEN}^{2,}$, Yung-Kuan $\mathrm{CHAN}^{3}$, Wei-Sheng $\mathrm{CHUNG}^{5}$ and Dah-Jye LEE $^{6}$}

${ }^{1}$ Department of Management Information Systems and 2Department of Dental Technology \& Materials Science, Central Taiwan University of Science and Technology, Taichung, 40601 Taiwan

${ }^{3}$ Department of Urology, Feng Yuan Hospital, Ministry of Health and Welfare, Taichung, 42055 Taiwan

${ }^{4}$ Department of Management Information Systems, National Chung Hsing University, Taichung, 40227 Taiwan

${ }^{5}$ Department of Internal Medicine, Taichung Hospital, Ministry of Health and Welfare, Taichung, 40343 Taiwan

${ }^{6}$ Department of Electrical Engineering, Brigham Young University, Provo, Utah 84602, USA

${ }^{*}$ Corresponding author: yfchen@ctust.edu.tw; ${ }^{\S}$ Authors with equal contribution

Keywords: Erectile Dysfunction, Chronic Disease, Decision Support System.

\begin{abstract}
Men with ED generally complain failure to attain or maintain an adequate erection during sexual activity. The prevalence of ED is strongly correlated with age, affecting about $40 \%$ of men at age 40 and nearly $70 \%$ at aged 70 . A variety of chronic diseases, including diabetes, hypertension, cardiovascular disease (CVD), chronic renal failure, depression, sleep disorder, and gout were shown to be associated with ED. In this study, the data used for designing the clinical decision support system (CDSS) was retrieved from a subset of the National Health Insurance Research Database (NHIRD) of Taiwan. The positive cases were male patients with age ranging from 20 to 65 years old who were diagnosed with ED (ICD-9-CM Code 607.84) between Jan. 2000 and Dec. 2010 confirmed by at least 3 outpatient visits, while the negative cases were randomly selected from the database with no history of ED and 1:1 frequency- and age-matched with the ED patients. Experiments of 1 cross validation (10 folds) and 2 independent training and testing (ITT) were conducted to verify the effectiveness of the predictive models. The results show that the sensitivity, specificity, and accuracy of tenfold cross validation achieved $69.45 \%, 69.45 \%$, and $69.54 \%$, respectively. For the ITT experiments, the sensitivity, specificity, accuracy, and the area under ROC curve were $70.28 \%, 72.48 \%, 71.32 \%$, and 0.7226 , respectively in the first experiment, and $69.42 \%$, $70.74 \%, 70.06 \%$, and 0.7143 , respectively, in the second experiment. Future works will focus on designing the CDSSs with ensemble classifiers consisting of multiple SVM models by adopting the laboratory data to improve the predictive performance for ED prediction.
\end{abstract}

\section{Introduction}

Erectile dysfunction (ED) affects millions of men worldwide [1]. Men with ED generally complain failure to attain or maintain an adequate erection during sexual 
activity. The prevalence of ED is strongly correlated with age, affecting about $40 \%$ of men at age 40 and the prevalence increases to nearly $70 \%$ in men aged 70 [2].

The incidence of ED was reported to increase with age; biological aging causes older men to be more likely to have comorbid conditions than younger men [3]. It was also reported that the prevalence of ED is positively associated with risk factors of cardiovascular disease (CVD) [4]. The objective of this study was to design a CDSS for predicting ED according to the demographic information of patients as well as the comorbidities and their acquired ages before ED diagnosis.

Chronic Diseases Associated with ED. A variety of chronic diseases, including diabetes, hypertension, cardiovascular disease (CVD), chronic renal failure, and depression, were also shown to be related to ED [5]. Recently, our studies showed that gout [6] and sleep disorder [7] were also strongly associated with ED. Ischemic heart disease is associated with ED, which in turn is deemed as an efficient predictor of ischemic heart disease [8]. It was reported that ED occurred in more than $60 \%$ of men with diabetes; while among the ED patients, $20.2 \%$ were found to have acquired diabetes [9].

Patients with congestive heart failure may be comorbid with ED for a variety of reasons, including atherosclerosis, neuropathy, hypogonadism, psychogenic factors, and medication side effects [10]. ED is generally observed to be comorbid with hypertension in men. among the 272325 patients with ED, $23.9 \%$ were found to be comorbid with hypertension [9]. Depression is also prevalent in patient with ED [9]. Regardless of treatment with sildenafil or placebo, a significant improvement in ED was found to be associated with a significant improvement in depression [11]. ED frequently occurs in patients with chronic renal failure (CKD); prevalence of ED in patients with CKD was $70 \%$ in average according to a meta-analysis study[12].

Recently, we have discovered that ED risk for patients with gout exhibited a 1.21-fold adjusted hazard ratio compared to those without gout based on the National Health Insurance Research Database (NHIRD) study [6]. Moreover, according to the nationwide population-based cohort study which examined 34,548 sleep disorder (SD) patients with a follow-up period of approximately 250,000 person-years, it was discovered that SD patients are at a 2.11 -fold increased risk of developing ED, compared with the general population [7].

Clinical Decision Support Systems. Clinical decision support systems (CDSSs) are promising in providing useful information and expert knowledge to enhance diagnostic performance and improve healthcare quality in clinical setting. It was reported that among the 97 proposed CDSS applications, including 10 diagnostic systems, 21 reminder systems, 37 disease management systems, and 29 drug-dosing or prescribing systems, $64 \%$ of the CDSSs demonstrated improved outcomes in medical practice [13]. Recently, we have conducted a prospective study to verify the effectiveness of the CDSS in ventilator weaning, capable of predicting weaning outcome and reducing duration of ventilator support for patients [14].

\section{Materials and Methods}

The National Health Research Institutes (NHRI) in Taiwan is authorized to establish the National Health Insurance Research Database (NHIRD), as well as to manage registration and claim data for the 23 million insurers. The data for designing the clinical decision support system was retrieved from a subset of the Taiwan NHIRD, consisting of 1 million randomly sampled beneficiaries enrolled in the NHI program. 
The NHI dataset includes information regarding medical facility registries, inpatient orders, ambulatory care, dental services, prescription drugs, and physicians providing services.

Data Retrieval. The positive cases were male patients with age ranging from 20 to 65 years old who were diagnosed with ED (ICD-9-CM Code 607.84) by physicians between Jan. 2000 and Dec. 2010 and were confirmed for at least 3 outpatient visits, while the negative cases were randomly selected from the database with no history of ED and 1:1 frequency- and age-matched with the ED patients. Patients with ED diagnosed before any comorbidities being diagnosed were excluded.

Data of 2311 positive cases (ED patients) and 2311 negative cases (non-ED patients) were used for designing the predictive models. Table 1 compares the demographic characteristics and comorbidity incidences between patients with and without ED. As shown in the table, there is no significant difference $(p=1)$ between 2 groups because of age-match control. On the other hand, patients with ED exhibited higher incidence rates of any types of comorbidities than the non-ED $(p<0.001)$. Regarding the ages of comorbidities being diagnosed, the ED patients presented significantly younger ages in acquiring depression and sleep disorder ( $p<0.05$ and $p<0.001$, respectively).

Feature Selection. Feature selection takes the advantage of reducing the number of features and the size of storage requirements, decreasing training and computational time, facilitating data visualization and understanding, and improving predictive performance. Recently, genetic algorithm (GA) was also proposed as a useful method for feature selection, and, sometimes, this strategy is also used for the adjustments of cost value and kernel parameter of SVM together with the selection of features when designing a classifier. Genetic algorithm was used to select the salient features and to adjust SVM parameters for designing the CDSSs.

Model Validation. SVM was applied to construct the CDSSs for predicting ED. Experiments, including 1 cross validation and 2 independent training and testing (ITT) were conducted to verify the effectiveness of the predictive models. In this study tenfold cross-validation was adopted for testing the CDSS predictive performance. The sensitivity, specificity, and accuracy were calculated for cross validation. Additionally, independent training and testing (ITT) was also used for validating the effectiveness of the CDSS [15]. In the 1st ITT experiment, data extracted from Jan. 2000 till Dec. 2005 were used for training the CDSS, whereas the data retrieved after Dec. 2005 till Dec. 2010 used for validation. For the $2^{\text {nd }}$ ITT experiment, the data extracted from Jan. 2000 till Dec. 2007 were used for training the CDSS, whereas the data retrieved afterward adopted for validation. The sensitivity, specificity, accuracy, and area under ROC curve (AUC) were used for evaluating the predictive performance. This study was approved by the Ethics Review Board of Chianan Psychiatric Center, Ministry of Health and Welfare (No. 15-021).

\section{Experimental Results}

The features selected using GA for designing the CDSSs include age, presence of diabetes, ischemic heart disease, congestive heart failure, hypertension, chronic renal failure, and gout, as well as the ages of diabetes, depression, gout, and sleep disorder diagnosed before ED outbreaks. Table 2 presents the performances of cross validation and ITT experiments of the CDSSs. As indicated in the table, the sensitivity, specificity, and accuracy of tenfold cross validation achieved 69.45\%, 69.45\%, and 69.54\%, respectively. Regarding the ITT experiments, the sensitivity, specificity, accuracy, and 
the area under ROC curve were $70.28 \%, 72.48 \%, 71.32 \%$, and 0.7226 , respectively for the first experiment, and $69.42 \%, 70.74 \%, 70.74 \%$, and 0.7143 , respectively, for the second experiment.

Table 1. Demographic characteristics and comorbidities in patients with and without ED.

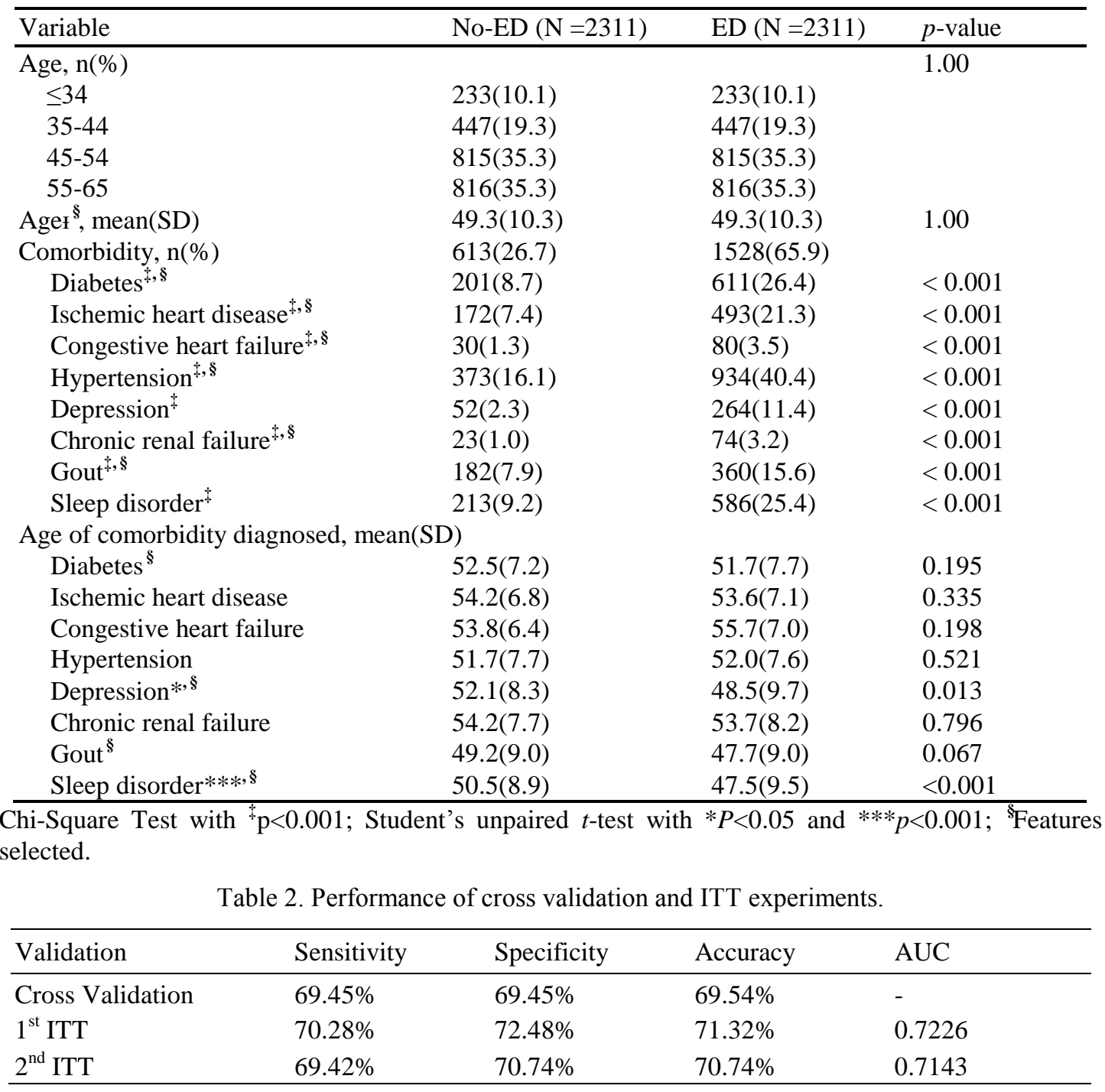

\section{Discussions and Conclusions}

The selected features for designing the CDSSs include age, presence of diabetes, ischemic heart disease, congestive heart failure, hypertension, chronic renal failure, and gout, as well as the ages when the diabetes, depression, gout, and sleep disorder diagnoses had been diagnosed. Interestingly, although the positive cases and the negative cases were age-matched without showing any significant difference $(p=1)$, age was still a feature selected for designing the CDSS. As reported recently in our age-matched study [6], the hazard ratio of ED increased with age for both gout and non-gout patients.

As indicated in Table 1, all the comorbidities exhibited highly significant difference between ED and non-ED groups $(p<0.001)$. However, depression and sleep disorder were not selected for CDSS design. Nonetheless, the statistical analyses of the acquired ages of these 2 diseases exhibited significant difference between ED and non-ED groups $(p<0.05$ and $p<0.001)$, and were selected as salient features using GA. On the 
other hand, the acquired ages of ischemic heart disease, congestive heart failure, hypertension, and chronic renal failure were not selected, but the presence of these diseases were selected for CDSS design. It mimics that presences and acquired ages of comorbidities might have enforcing and compensatory effects in improving predictive performance of the CDSSs.

Although the predictive performance of the designed systems presented here is fair, it is the first CDSS presented to predict the occurrence of ED. Nobody has ever introduced a CDSS for ED into clinical setting. More clinical information and lab test data are useful for increasing the predictive performance of the CDSSs [14, 16]. Hsu et al. [14] reported that demographic information, physiological and disease variables, and care and treatment factors were demonstrated to be effective in predicting successful ventilator weaning with a sensitivity as high as $87.7 \%$. Luo et al. [16] argued that lab test data were expected to help increase the accuracy of predictive models for bronchiolitis. The lab test data of the NHIRD adopted in this study are not available currently, making the predictive performance of designed CDSSs greatly compromised.

An ensemble classifier which combines multiple classifiers often obtains better predictive performance than a single-kernel classifier. In our previous study, the ensemble SVM classifier, consisting of three SVM models, was designed for bridge classification and prediction of the disulfide bonding pattern, achieving an accuracy of $96.5 \%$ and $89.2 \%$, respectively [17]. In the future, designing CDSSs for ED prediction will focus on adopting laboratory data to improve the predictive performance. In addition, ensemble classifiers consisting of multiple SVM models will be designed to improve the predictive performance.

\section{Acknowledgements}

This study was supported by Ministry of Science and Technology of Taiwan (Grant No. MOST104-2410-H-166-005) and Fulbright Foundation for Scholarly Exchange.

\section{References}

[1] P. Grant, G. Jackson, I. Baig and J. Quin. Erectile dysfunction in general medicine. Clin Med (Lond), 13(2) (2013). 136-140.

[2] H. A. Feldman, I. Goldstein, D. G. Hatzichristou, R. J. Krane and J. B. Mckinlay. Impotence and Its Medical and Psychosocial Correlates - Results of the Massachusetts Male Aging Study. Journal of Urology, 151(1) (1994). 54-61.

[3] E. Selvin, A. L. Burnett and E. A. Platz. Prevalence and risk factors for erectile dysfunction in the US. Am J Med, 120(2) (2007). 151-157.

[4] T. Y. Ahn, J. K. Park, S. W. Lee, J. H. Hong, N. C. Park, J. J. Kim, K. Park, H. Park and J. S. Hyun. Prevalence and risk factors for erectile dysfunction in Korean men: results of an epidemiological study. J Sex Med, 4(5) (2007). 1269-1276.

[5] A. E. Weinberg, M. Eisenberg, C. J. Patel, G. M. Chertow and J. T. Leppert. Diabetes severity, metabolic syndrome, and the risk of erectile dysfunction. J Sex Med, 10(12) (2013). 3102-3109.

[6] Y. F. Chen, H. H. Lin, C. C. Lu, C. T. Hung, M. H. Lee, C. Y. Hsu and W. S. Chung. Gout and a Subsequent Increased Risk of Erectile Dysfunction in Men Aged 64 
and Under: A Nationwide Cohort Study in Taiwan. Journal of Rheumatology, 42(10) (2015). 1898-1905.

[7] H. H. Lin, F. M. Ho, Y. F. Chen, C. M. Tseng, C. C. Ho and W. S. Chung. Increased risk of erectile dysfunction among patients with sleep disorders: a nationwide population-based cohort study. International Journal of Clinical Practice, 69(8) (2015). 846-852.

[8] C. Gazzaruso, S. Giordanetti, E. De Amici, G. Bertone, C. Falcone, D. Geroldi, P. Fratino, S. B. Solerte and A. Garzaniti. Relationship between erectile dysfunction and silent myocardial ischemia in apparently uncomplicated type 2 diabetic patients. Circulation, 110(1) (2004). 22-26.

[9] A. D. Seftel, P. Sun and R. Swindle. The prevalence of hypertension, hyperlipidemia, diabetes mellitus and depression in men with erectile dysfunction. Journal of Urology, 171(6) (2004). 2341-2345.

[10] E. R. Schwarz, S. Rastogi, V. Kapur, N. Sulemanjee and J. J. Rodriguez. Erectile dysfunction in heart failure patients. Journal of the American College of Cardiology, 48(6) (2006). 1111-1119.

[11] S. N. Seidman, S. P. Roose, M. A. Menza, R. Shabsigh and R. C. Rosen. Treatment of erectile dysfunction in men with depressive symptoms: Results of a placebo-controlled trial with sildenafil citrate. American Journal of Psychiatry, 158(10) (2001). 1623-1630.

[12] S. D. Navaneethan, M. Vecchio, D. W. Johnson, V. Saglimbene, G. Graziano, F. Pellegrini, G. Lucisano, J. C. Craig, M. Ruospo, G. Gentile, V. M. Manfreda, M. Querques, P. Stroumza, M. Torok, E. Celia, R. Gelfman, J. N. Ferrari, A. Bednarek-Skublewska, J. Dulawa, C. Bonifati, J. Hegbrant, C. Wollheim, E. A. Jannini and G. F. M. Strippoli. Prevalence and Correlates of Self-Reported Sexual Dysfunction in CKD: A Meta-analysis of Observational Studies. American Journal of Kidney Diseases, 56(4) (2010). 670-685.

[13] A. X. Garg, N. K. J. Adhikari, H. McDonald, M. P. Rosas-Arellano, P. J. Devereaux, J. Beyene, J. Sam and R. B. Haynes. Effects of computerized clinical decision support systems on practitioner performance and patient outcomes - A systematic review. Jama-Journal of the American Medical Association, 293(10) (2005). 1223-1238.

[14] J. C. Hsu, Y. F. Chen, W. S. Chung, T. H. Tan, T. S. Chen and J. Y. Chiang. Clinical Verification of A Clinical Decision Support System for Ventilator Weaning. Biomedical Engineering Online, 12(2013).

[15] Y. F. Chen, P. C. Huang, K. C. Lin, H. H. Lin, L. E. Wang, C. C. Cheng, T. P. Chen, Y. K. Chan and J. Y. Chiang. Semi-Automatic Segmentation and Classification of Pap Smear Cells. IEEE Journal of Biomedical and Health Informatics, 18(1) (2014). 94-108.

[16] G. Luo, F. L. Nkoy, P. H. Gesteland, T. S. Glasgow and B. L. Stone. A systematic review of predictive modeling for bronchiolitis. International Journal of Medical Informatics, 83(10) (2014). 691-714.

[17] H. H. Lin, J. C. Hsu, Y. N. Hsu, R. H. Pan, Y. F. Chen and L. Y. Tseng. Disulfide connectivity prediction based on structural information without a prior 
knowledge of the bonding state of cysteines. Computers in Biology and Medicine, 43(11) (2013). 1941-1948. 